\title{
Ökonomie der Intimität: Racine, Rousseau
}

Ô mon Fils,

que tes jours coûtent cher à ta Mère! ${ }^{1}$

\section{Vorüberlegung: Zum Begriff der Ökonomie der Intimität}

Wenn man Joseph Vogls fraglos maßgebliche Arbeit Kalkül und Leidenschaft ${ }^{2}$ befragt, ist die bürgerliche, die moderne Affektökonomie über folgende Eckwerte zu bestimmen: (1) Affekte werden in zunehmendem Maße berechenbar, indem man sie einem Kalkül (ökonomischer Steuerung) unterwirft. (2) Von Affektökonomie ist erst dann zu reden, wenn ein hinlänglich epistemologischer Begriff von Ökonomie ausgeprägt ist. Vogl zufolge ist der historische Bruch, der im 17./18. Jahrhundert statthat, deshalb nicht länger mit anthropologischen Konzepten zu fassen. Zur Verdeutlichung verweist Vogl auf die diffuse Ökonomie von >Naturvölkern`, in denen der Zugriff auf Güter durch ein Amalgam aus Verwandtschaftsbeziehungen und Nutzungs-/Genussrechten an Häusern und Grundstücken geregelt ist. Angesichts solcher Verhältnisse dekretiert er:

\footnotetext{
Was aber fehlt, oder besser: was ganz einfach nicht existiert, ist ein ökonomischer Akt im strengen Sinn, was fehlt, sind Dinge, Handlungen und Verhaltensweisen, die sich gerade deshalb zusammenfügen und aufeinander beziehen, weil sie ökonomische sind. ${ }^{3}$
}

So besehen wäre der Begriff der Ökonomie nur unter den Bedingungen einer im Grundansatz ökonomistischen Episteme angemessen - denn wie anders ist ein ökonomischer Akt zu interpretieren, in dem sich Dinge, Handlungen, Verhaltensweisen aufeinander beziehen, weil sie >ökonomische` sind. So schlüssig der Ansatz von Kalkül und Leidenschaft in sich ist, er ist meines Erachtens in folgenden Punkten zu hinterfragen:

1 Jean Racine: Andromaque, III, 8, v. 1050. In: Ders.: Euvres complètes. Bibliothèque de la Pléiade. Bd.1. Herausgegeben von Georges Forestier. Paris: Gallimard 1999, S.193-256.

2 Joseph Vogl: Kalkül und Leidenschaft. Poetik des ökonomischen Menschen. Zürich: Diaphanes (2002) ${ }^{4} 2011$.

3 Ebda., S. 11. 
Methodisch: Offensichtlich versucht Vogl den Begriff der Ökonomie für den eigenen Ansatz nachgerade zu monopolisieren. Dem ist entgegenzuhalten: So divers die Tauschstrukturen etwa von `Naturvölkern ‘ auch sind, sie ermöglichen ihnen immerhin einen geregelten Tausch von Gütern. Insofern ist diesen Ethnien nicht abzusprechen, dass auch sie eine Ökonomie besitzen. Denn ökonomisch sind gesellschaftliche Beziehungen bereits dann, wenn sie aufgrund welchen Regelwerks auch immer den Besitz und Tausch von Gütern gewährleisten können. Mit anderen Worten: Als ökonomisch sind alle gesellschaftlichen Beziehungen anzusehen, die Tauschakte betreffen. Diese sind zu unterscheiden von dem, was Vogl als »ökonomischen Akt» bezeichnet. ${ }^{4}$

Sachhaltig/historisch: Unter dem Stichwort von Leidenschaft/Liebe und Freundschaft entstehen im 18. Jahrhundert soziale Konzepte, die sich nicht nur gegen die Feudalordnung wenden, sondern ebenso sehr gegen den Ökonomismus des aufstrebenden Bürgertums. Im Gefolge dieser polaren Festlegung ist die bürgerliche Gesellschaft zutiefst gespalten. Während sie auf der Ebene von Güterproduktion und -tausch strikt ökonomisch operiert, lehnt sie auf interpersoneller Ebene Tauschbeziehungen kaum weniger strikt ab. Dieser Widerspruch von Ökonomismus und Antiökonomismus reicht herauf bis in die Jetztzeit. Man denke im wirtschaftswissenschaftlichen Umfeld an die Kontroverse von (neo-) liberalen und sozial orientierten Wirtschaftsmodellen (die im Grundansatz auf das intime Konzept der fraternité zurückverweisen). Er trägt sich in anderer Weise

\footnotetext{
4 Da Vogls Ansatz auf einem historisch-epistemologischen Konzept aufruht, wäre es m. E. angemessener, statt von einem ökonomischen Akt von einer ökonomischen Praktik - Praktik im Sinne Foucaults - zu reden. Das Konzept des Aktes erfährt bekanntlich im Gefolge der Phänomenologie, aber auch im Gefolge der Sprechakttheorie eine beträchtliche Konjunktur. Foucault schließt hieran an, nicht ohne diese Konzepte zu verschieben. Unter Praktiken versteht Foucault keine einzelnen, isolierten Akte (die nichtsdestoweniger eine komplexe Wahrnehmungs- oder Interaktionskonstellation betreffen können), sondern ein historisches Ensemble von - eben - Praktiken. Gemessen an diesem komplexen historischen Ansatz ist der Begriff des Tauschakts - oder auch nur der des Tauschs - historisch weniger filigran, sondern vorneweg heuristisch ausgerichtet. Er eröffnet gerade deshalb eine breitere Vergleichsbasis. Vgl. hierzu, im Unterschied zu Vogl, die Klassiker der anthropologisch/ethnologisch fundierten Tauschtheorie: Marcel Mauss: Essai sur le don. Forme et raison de l'échange dans les sociétés archaïques. Paris: PUF (1950) 22012, Claude Lévi-Strauss: Les structures élémentaires de la parenté. Berlin/New York: Mouton de Gruyter (1949) ${ }^{2} 1967$ sowie neuerdings David Graeber: Schulden. Die ersten 5.000 Jahre. Aus dem Amerikanischen von Ursel Schäfer/Hans Freundl u. a. Stuttgart: Klett-Cotta (12011) ${ }^{72012 . ~ Z u m ~ N e x u s ~ v o n ~}$ Schuld und Schulden, vgl. bereits früher Franziska Sick: Tragisches Potential und untragisches Ende. Absolutistische Konzepte in den frühen Dramen Corneilles. In: Rudolf Behrens/Roland Galle (Hg.): Historische Anthropologie und Literatur. Romanistische Beiträge zu einem neuen Paradigma der Literaturwissenschaft. Würzburg: Königshausen \& Neumann 1995, S.91-107, und hierbei insbesondere die Unterscheidung von `Schuldigsein an` und `Schulden haben bei`, S. 106-107.
} 
im ästhetischen Bereich ab. Während Vogl Affektökonomie als epistemologische Konstellation versteht, zu der es kein Außen gibt, unterstellt man anderweitig, dass Affekte/Kunst eine Gegenmacht gegen den schieren Ökonomismus des Kapitalismus bilden können. ${ }^{5}$ Typischerweise betrachtet solche regelmäßig humanitäre Politästhetik nur gezähmte, empathische Affekte, nicht jedoch die Gier der Finanzindustrie.

So diametral diese Lesart der Vogl'schen zuwiderläuft - beide besitzen eine im Kern unhinterfragte Gemeinsamkeit: Sie tragen die Beziehung von Affekt und Ökonomie auf der Achse von Individuum und Gesellschaft ab. Ausgeblendet bleibt hierbei, dass das moderne Konzept der Intimität einen Beziehungstyp und mit ihm ein Affektmodell inauguriert, das sich als dezidiert außergesellschaftlich versteht. Es propagiert eine affektive Nahbeziehung zwischen Zweien, und das heißt: eine Beziehung unter Ausschluss der Gesellschaft. Wenn dergestalt Affekte erklärtermaßen zwischen Zweien zu >tauschen` wären, muss es vorneweg irritieren, dass die Affektökonomi $\mathrm{e}^{[\mathrm{M}] 6}$ sie in dem Spannungsfeld von Individuum und Gesellschaft zu verorten versucht. Hiervon ausgehend ist der unterschiedliche Grundansatz einer Affektökonomie ${ }^{[\mathrm{M}]}$ und einer Ökonomie der Intimität ${ }^{[\mathrm{M}]}$ genauer zu bestimmen. Während zumal die Vogl'sche Affektökonomie ${ }^{[\mathrm{M}]}$ die Affekte in Funktion zu gesellschaftlichen Normen und Wissenssystemen setzt, akzentuiert eine Ökonomie der Intimität den zwischenmenschlichen Beziehungsaspekt. Intimität ist hierbei nicht mit Affektivität oder gar sexuellen Intimitäten zu verwechseln. Sie meint den vergleichsweise formalen Tatbestand, dass zwei Interaktionspartner ohne Bezugnahme auf gesellschaftliche Werte zu interagieren - und das heißt immer auch: zu tauschen - versuchen. Dieser Interaktionstyp trifft auf die moderne Liebe zu, aber auch auf das Konzept der Freundschaft, das in ihrem Gefolge entsteht.

Im Ergebnis könnte der Befund beider Ansätze kaum konträrer sein. Während die Affektökonomie ${ }^{[\mathrm{M}]}$ postuliert, dass der `ökonomische Aktı das ältere Modell eines Amalgams von interpersonellen Verpflichtungen und Gütertausch ablöst, vertritt die Ökonomie der Intimität ${ }^{[\mathrm{M}]}$ die Auffassung, dass interpersonelle Verbindlichkeiten nach wie vor fortbestehen. Zugrunde liegen hierbei konträre Geschichtsmodelle. So kapitalismuskritisch sich Vogl zur historischen Entwick-

5 Man denke hierbei etwa an neomarxistische Positionen oder aber auch aktueller Weise an die Documenta 14, die in breitem Umfang auf diese zurückgreift.

6 Die Termini Affektökonomie und Ökonomie der Intimität sind insofern ambig, als sie sowohl einen methodischen, modellhaften Ansatz als auch einen historischen Sachverhalt benennen. Um diese Zweideutigkeit zu vermeiden und das heißt, um das explanans vom explanandum zu trennen, indiziere ich den methodischen Aspekt mit Affektökonomie ${ }^{[M]}$ bzw. Ökonomie der Intimität $^{[\mathrm{M}]}$. Wenn die beiden Begriffe nicht indiziert sind, bezeichnen sie den historischen Sachverhalt. 
lung auch stellt, er trägt sie auf der Achse eines Fortschrittsmodells ab, demzufolge ein älteres System durch ein neueres, leistungsfähigeres funktionell beerbt wird. Eine Ökonomie der Intimität ${ }^{[\mathrm{M}]}$ wird an dieser Stelle anders argumentieren wollen: Neue Systeme beerben nicht alte, sondern sie differenzieren sie, indem sie sie in Subsysteme aufspalten ${ }^{7}$ oder aber auch nur bestehende Systemstellen neu besetzen.

Die Entwicklung im 18. Jahrhundert ist als solcher Differenzierungsprozess $\mathrm{zu}$ verstehen. Während man dazu übergeht, gesellschaftliche Beziehungen rein wirtschaftlich zu kodieren, entwickelt man im Gegenzug ein Modell der Intimität, der Gegengesellschaftlichkeit, das dezidiert antiökonomisch ist und nichtsdestoweniger auf Tauschstrukturen basiert. Formelhaft fassbar wird diese Differen-

7 Das ist bekanntlich ein Kernargument der Systemtheorie: dass in dem Maße, in dem ein Unterschied einen Unterschied macht, Systeme sich differenzieren, sich in Teilsysteme aufspalten; vgl. hierzu ursprünglich Gregory Bateson: Ökologie des Geistes. Anthropologische, psychologische, biologische und epistemologische Perspektiven. Übersetzt von Hans Günter Holl. Frankfurt a. M.: Suhrkamp ( $\left.{ }^{1} 1981\right){ }^{5} 1983$ und später Niklas Luhmann: Soziale Systeme. Grundriss einer allgemeinen Theorie. Frankfurt a. M.: Suhrkamp 1984. Zu einer systemtheoretischen Interpretation von Intimität, vgl. insbesondere Niklas Luhmann: Liebe als Passion. Zur Codierung von Intimität. Frankfurt a. M.: Suhrkamp ( $\left.{ }^{1} 1982\right){ }^{13} 2015$ und ders.: Liebe. Eine Übung. Herausgegeben von André Kieserling. Frankfurt a. M.: Suhrkamp ('2008) 42014. - Ohne dass ich diesen Punkt allzu sehr vertiefen will, ist darauf hinzuweisen, dass meine Ergebnisse in nicht unbeträchtlichem Maße von Luhmanns Studie abweichen. Das betrifft zum einen die Datierung: Liebe, Intimität gibt es m. E. bereits vor dem 18. Jahrhundert, genauer: ab Racine, und dies in durchaus verschärfter, zugespitzter Form. Das betrifft zum anderen die methodischen Bordmittel: Auch wenn es da und dort hilfreich ist, auf den Werkzeugkasten der Systemtheorie zurückzugreifen - die methodische Leitkategorie der vorliegenden Studie ist ein von ethnologischen/ anthropologischen Ansätzen inspirierter Begriff des Tauschs. Deshalb vertritt sie, wenn man so will, einen vergleichsweise zurückhaltenden ssystemtheoretischen` Ansatz. Während Luhmanns Systemtheorie die Emergenz von Systemen in den Vordergrund stellt, unterstelle ich, dass es methodisch durchaus fruchtbar sein kann, ein wie auch immer vage zu bestimmendes statisches Set von (anthropologischen) Systemstellen vorauszusetzen. Ein gutes Beispiel hierfür ist die Tauschkategorie. Und dort das Argument, dass man nicht ınicht tauschen` kann. So besehen vertrete ich sowohl gegenüber Vogl als auch gegenüber Luhmann eine vergleichsweise gemäßigte, mittlere Position. Während Vogl auf eine Emergenz der Ökonomie setzt, setzt Luhmann auf eine Emergenz der unwahrscheinlichen Kommunikation. Während Vogl zufolge in und mit der Affektökonomie ökonomisches Denken erst zu sich kommt, weil das ökonomische Denken universal wird und selbst den Affekt überbordet, entsteht Luhmann zufolge mit der Liebe als Passion eine völlig neue Beziehungsstruktur oder aber auch ein völlig neues gesellschaftliches Subsystem jenseits aller gesellschaftlichen Normen und Ökonomien. Ich neige eher der Luhmann'schen Auffassung zu, wobei ich dafürhalte, dass das neue System, so sehr es sich auch gegen die Ökonomie und gesellschaftliche Normen wendet, eben nicht ınicht tauschen` und ebenso wenig sich des Begriffs der Schuld entledigen kann. 
zierung, wenn man die negative Seite beider Tauschstrukturen betrachtet. Wenn Schulden typischer Weise eine monetäre, materielle Verpflichtung bezeichnen, und Schuld eine persönliche, so lässt sich der im 17./18. Jahrhundert erfolgende Wandel als eine Neuverteilung von Schuld und Schulden interpretieren. ${ }^{8}$ Der neuen Ordnung zufolge sind Schulden eine ausschließlich gesellschaftliche Angelegenheit und ist Schuld - wenn man von veritablen Straftaten einmal absieht - eine vorrangig intime.

Insofern moderne Intimität die interpersonelle Beziehung von Dank und Schuld beerbt, ist auch sie von Tauschakten geprägt, so sehr sie auch in der Eigenwahrnehmung und -darstellung darauf insistiert, dass Liebe ein unverfügbarer Affekt und deshalb nicht tauschbar sei. Dem ist entgegenzuhalten, dass man nicht >nicht tauschen « kann, genauso wenig wie man nicht `nicht kommunizieren $\triangleleft$ kann. Es ist deshalb kein Zufall, dass zumal die frühen literarisch modellierten Intimbeziehungen einen schuldhaften Verlauf nehmen, so namentlich in den Tragödien Racines und, etwas sozial vermittelter, bei Rousseau (Julie, ou La Nouvelle Héloïse) im Tod von Julie und deren Mutter. In solchen Handlungsverläufen tritt die paradoxe Grundkonstruktion der Ökonomie der Intimität zutage. In dem Maße, wie diese postuliert, dass Liebe nicht tauschbar ist, tendiert deren Wert gegen unendlich. Der Preis der Liebe ist deshalb das Äußerste, und das ist der Tod.

Benannt ist damit nicht zuletzt eine weitere Differenz zwischen Vogls Affektökonomie ${ }^{[\mathrm{M}]}$ und der im Folgenden zu entwickelnden Ökonomie der Intimität ${ }^{[\mathrm{M}]}$. Während die Affektökonomie ${ }^{[\mathrm{M}]}$ unterstellt, dass Affekte nach Maßgabe eines verbesserten gesellschaftlich-ökonomischen Modells regulierbar sind, vertritt die Ökonomie der Intimität ${ }^{[\mathrm{M}]}$ die Auffassung, dass sie hochgradig dereguliert sind. Weil man mit Liebe etwas zu tauschen versucht, das erklärtermaßen nicht tauschbar ist, verhält sich die Ökonomie der Intimität so hyperinflationär wie jedes andere ökonomische System, in dem die Gültigkeit von Tauschwerten problematisch wird.

Nicht auszublenden ist hierbei, welche Spannbreite inflationäre Ökonomien historisch einnehmen. Unter dem Titel L'échange symbolique et la mort entwickelt Jean Baudrillard eine postmoderne Ökonomie, in der sich Signifikant und Signifikat, Tauschwert und Gebrauchswert entkoppeln. ${ }^{9}$ Man tauscht hierbei nicht länger etwas, sondern nur noch Signifikanten. Vogl verzeichnet eine vergleichbare Entkopplung ab Goethe, Novalis. Die in unserem Zusammenhang zu entwickelnde Ökonomie der Intimität liegt vor dieser Zäsur. Man versucht in ihr gleichsam das Signifikat gegen das Signifikat zu tauschen.

8 Zur Unterscheidung von Schuld und Schulden, vgl. David Graeber: Schulden, S. 62-66.

9 Jean Baudrillard: L'échange symbolique et la mort. Paris: Gallimard 1976. 
Diese Beziehung wird nachstehend anhand folgender Eckpunkte zu entwickeln sein: Mit am radikalsten hat Racine die Auffassung >vorgetragen`, dass Liebe/Leidenschaft nicht tauschbar ist. Hieraus resultiert ein zutiefst instabiles System, in dem der Vorwurf des ingrat und monströse Gaben der Liebe so unverhofft wie unvermittelbar aufeinandertreffen. Rousseau setzt auf dieses Konzept intimer Interaktion in unterschiedlicher Weise auf. Während er in den Confessions in ihm befangen ist und es reproduziert, versucht er es in La Nouvelle Héloïse sozial zu vermitteln, nicht jedoch, indem er sich primär affektökonomischer Konzepte bedient, sondern indem er in abgewandelter Form auf tradierte interpersonelle Tauschkategorien wie die der Ehre und der Familie zurückgreift.

\section{Corneille: Cinna}

Obwohl mit Racine und Rousseau ein vergleichsweise großer historischer Bogen im Blick steht, lohnt es, einen Schritt zurückzutreten. Denn nur im Kontrast zum alten System kann das neue Kontur gewinnen. Man muss hierbei nicht bis zu den >Naturvölkern zurückgehen: In Corneilles Cinna ou la clémence d'Auguste ${ }^{10}$ ist Emilie bereit, Cinna zu heiraten, wenn er ihren Vater rächt, der von Augustus während seines Staatsstreichs ermordet wurde. Augustus seinerseits versucht Emilie und Cinna für sich zu gewinnen und zu versöhnen, indem er sie mit Gunstbeweisen und Gaben der Milde überhäuft. So will er unter anderem Cinna Emilie zur Frau geben.

So konträr beide Hochzeitsangebote sind, in beiden Fällen ist die Gabe, die Emilie ist oder zu geben hat, auf ein Tauschsystem bezogen. Das ist im einen Fall ein System der Clans und der Blutrache und im anderen Fall ein System des Staates und der Milde. Corneilles Cinna verhakt beide Systeme miteinander. Das Stück durchläuft hierbei eine mehrstufige Transaktion, in der Schuld und Schulden auf unterschiedlichen Niveaus beglichen und transformiert werden. Es handelt sich im Einzelnen um folgende Schritte: Augustus ist schuldig, da er Emilies Vater ermordete. Er versucht seine Schuld durch Milde, und das heißt durch gesellschaftliche Gaben, abzutragen. Man könnte auch sagen, er versucht sich von seiner Schuld freizukaufen. Emilie akzeptiert dieses Wiedergutmachungsangebot nicht und fordert nach wie vor Rache. Als sie und Cinna einen Staatsstreich gegen Augustus planen, werden sie ihrerseits schuldig. Am Ende

10 Pierre Corneille: Cinna ou la clémence d'Auguste. In: Ders.: Euvres complètes. Bibliothèque de la Pléiade. Bd.1. Herausgegeben von Georges Couton. Paris: Gallimard 1980, S.903-969. 
dieser (ökonomischen) Transaktionskette ist Augustus nicht nur entschuldet, sondern hat sich überdies das gesamte Schuldkapital angeeignet: Da er den beiden Verschwörern selbst noch den Staatsstreich und persönlichen Verrat verzeiht, stehen sie doppelt in seiner Schuld: aufgrund seiner anfänglichen Milde und aufgrund der erneuten Begnadigung.

Bemerkenswert ist in unserem Zusammenhang, in wie hohem Maße Corneille auf traditionelle Tauschstrukturen zurückgreift, um sie im Sinne des Absolutismus zu verschieben. In einem System der Clans sind es Familien, Sippen, die Frauen tauschen. Sie schaffen damit so etwas wie ein `Feudalsystem ‘ familialer Allianzen. Wie anhand von Cinna und Emilie zu besichtigen ist, können sie auf dieser Basis ein Widerstandsnest gegen übergeordnete zentralstaatliche Instanzen bilden. Der Corneille'sche Augustus unterläuft diese Struktur. Er vollzieht nicht nur eine Tauschoperation auf der Ebene des Frauentauschs, sondern transformiert diese im Sinne des Absolutismus. Cinna, so endet das Stück, erhält Emilie nicht aus ihrer eigenen Hand - und das wäre die Hand ihres Clans -, sondern aus der Hand des Augustus. Damit ist ein grundsätzlicher Systemwechsel im Sinne des Absolutismus eingeleitet. Vollends absolut sind Herrscher erst dann, wenn sie anstelle der Clans über die Gabe der Frau verfügen. Hierin besteht, wenn man so will, der zweite `Staatsstreich freiheitsliebende Rom, sondern in einem zweiten Schritt auch die Clans und die Gesetze der Verwandtschaft, aus denen diese ihre Macht beziehen. So wandelfähig können alte Systeme sein. Wenn auch nicht der moderne Territorialstaat in toto, so doch dessen Ideologie beruht darauf, dass Frauen nicht länger Gabe der Clans, sondern die Gabe des Königs sind.

Die Ebene der Affektivität - Emilie liebt Cinna - nimmt in diesem Tauschgeschäft trotz des vielbesprochenen Konflikts von amour et devoir eine vergleichsweise untergeordnete Rolle ein. Man sieht dies bereits daran, dass die Liebe Emilies als amour-estime einen wohldefinierten Preis hat. Vollends wenn man die Gesamtanlage des Stückes betrachtet, zeigt sich, dass die Liebe, dass der Affekt in Cinna kaum mehr als eine Deckschicht ist. In dem Maße, wie Corneille die Liebe zwischen Cinna und Emilie in den Vordergrund spielt, überblendet er den politischen Konflikt durch einen privaten. In etwas fragwürdiger Weise wird Cinna hierbei zu einem politischen Widerstandskämpfer aus Liebe. Ansatzweise tragfähig ist seine Position, weil sie sich (noch) auf einen Wertekanon berufen kann, in dem im amour-estime Ehre und Liebe genauso ungeschieden sind wie Schuld und Schulden: Cinna könnte Emilie heiraten, wenn er sie rächt, das ist eine Frage von persönlicher Schuld und Verpflichtung. Cinna ist Augustus aufgrund seiner Milde verpflichtet, das sind Schulden in einem mehr oder minder materiellen Sinne. Auch wenn man beide Seiten noch zu verrechnen versucht, sie treten ansatzweise bereits auseinander. Geschuldet ist diese Dissoziierung 
dem Umstand, dass Corneille mit dem Absolutismus eine gesellschaftliche Abstraktion einführt, die über den Gesetzen der Clans steht. Im Gegenzug werden deren Gesetze und Racheansprüche tendenziell zu einer Privatangelegenheit. So besehen ist die moderne Liebe die Kehrseite des Absolutismus, so besehen propagiert der Konflikt von amour et devoir in ersten Ansätzen die moderne Trennung von intimer Schuld und öffentlichen Schulden.

Racine greift diese erste Differenzierung auf, indem er sie auf den Kopf stellt und damit zugleich radikalisiert. Während Corneille die Schulden des Helden gegenüber dem Staat ins Zentrum rückt, verabsolutiert Racine den Affekt - und mit ihm die intime Tauschbeziehung und Schuld. Im Zuge dieser Umbesetzung werden gegenbildlich $\mathrm{zu}$ Corneille gesellschaftliche Verpflichtungen peripher und die Racine'schen Herrscher und Heroen auf die Rolle des staatsvergessenen, leidenschaftlich Liebenden `reduziert`. Von einer Ökonomie der Intimität ist erst nach Maßgabe dieser Umbesetzung und Reduktion zu reden.

\section{Racine: Andromaque}

Anders als in Cinna stiftet die Hochzeit in Racines Andromaque keine Allianz, sondern führt zu einem Blutbad. Wobei sich die Frage stellt, ob diese Bluthochzeit etwas jenseits aller Ökonomie, ein schieres Wüten der Leidenschaft oder eine andere Form von in diesem Fall intimer und deshalb deregulierter >Affektı-Ökonomie ist.

Eine weitgehend nicht ökonomische Haltung nimmt Andromaque zu Beginn des Stückes ein. Nachdem Achill vor Troja ihren Gatten Hector ermordet hat, fallen sie und ihr Sohn Astyanax Pyrrhus als Kriegsbeute zu. Trotz der Gräuel vor Troja will Andromaque sich nicht rächen. Sie begnügt sich mit dem, was ihr verblieben ist: Astyanax. Sie will ihn sich bewahren, zumal Astyanax für sie nicht nur ihr Sohn, sondern auch das Abbild Hectors ist. Andromaque nimmt damit eine sehr bescheidene Position ein. Sie erscheint wie aus der Gesellschaft und ihren Tausch-, Blutrache- und Heiratsbeziehungen herausgefallen. Als ihr Pyrrhus anbietet, er würde, so sie ihn heiratet, Troja wieder aufbauen und ihren Sohn als König einsetzen, antwortet sie ihm:

Seigneur, tant de grandeurs ne nous touchent plus guère, Je les lui promettais tant qu'a vécu son Père. ${ }^{11}$

11 Jean Racine: Andromaque, I, 4, v. 333-34. 
Im selben Geiste bittet sie später Pyrrhus darum, ihr und ihrem Sohn Asyl zu gewähren. Obwohl - oder aber auch weil - Andromaque die wohl defensivste Figur im Werk Racines ist, ist bereits an ihr ablesbar, dass wir mit ihr in eine Tauschordnung eintreten, die grundsätzlich anders strukturiert ist als noch die Corneille'sche. Anders als Emilie will Andromaque weder Rache für den ermordeten Anverwandten noch die Restitution eines Reiches. Sie will einen Schutzraum für ihren Sohn und für ihre Trauer.

Gemessen hieran erweist sich alles, was Pyrrhus Andromaque an gesellschaftlichen Gaben anzubieten hat, als uneigentliche Gabe. Deshalb versucht er zugleich auf einer weiteren Ebene zu tauschen. Noch bevor die Griechen den Kopf von Astyanax fordern, noch bevor er Andromaque anbietet, Troja wiederaufzubauen, ${ }^{12}$ klingt diese zweite Tauschebene an: Pyrrhus hat Astyanax weggesperrt. Er verweigert Andromaque weitgehend den Zutritt zu ihrem Sohn und droht ihr, sofern sie ihn, Pyrrhus, nicht heiratet, Astyanax zu ermorden. Abgesehen davon, dass dies eine platte Erpressung ist, versucht Pyrrhus sich die Liebe Andromaques zu serkaufen`, indem er ihr den Geliebten gibt oder aber auch verweigert, das heißt mit anderen Worten: Er versucht in wie vager Weise auch immer Liebe gegen Liebe $\mathrm{zu}$ tauschen. Auch wenn dieser Term angesichts der offenen Drohungen des Pyrrhus etwas irritieren mag - was Pyrrhus Andromaque anbietet, ist nicht länger ein gesellschaftlich kodierter, sondern ein intimer Tausch. Intim ist dieser Tausch, weil er die Gabe der Frau nicht länger gegen einen gesellschaftlichen Wert wie das Reich oder den Thron zu tauschen versucht, sondern gegen Liebe oder, dinglicher gefasst, gegen den Geliebten.

Selbstredend ist das Tauschmodell, mit dem Racine an dieser Stelle aufwartet, äußerst konstruiert. Pyrrhus kann Andromaque den >Geliebten` nur geben, weil dieser nur dessen Platzhalter ist. Astyanax ist zwar Hectors Sohn, aber er ist nicht Hector. So sachlich vorweisend dieses Szenario ist, so sehr es die schiere Gabe der Liebe unter Ausschluss gesellschaftlicher Tauschwerte inauguriert, in struktureller Hinsicht ist es nichtsdestoweniger vergleichsweise traditionell gefasst. Einfach deshalb, weil in ihm mit Astyanax noch ein Vermittlungsglied, eine konkrete Gabe oder aber auch so etwas wie eine Münze zirkuliert. Diese Münze, Astyanax, ist allerdings ein äußerst ambivalentes Zahlungsmittel. Sie ist zugleich die Sache selbst und sie ist sie nicht. Astyanax ist das Wertvollste, das Andromaque besitzt, deshalb kommt er für Pyrrhus als Zahlungsmittel in Betracht, er ist aber auch die Postfiguration Hectors und das heißt so etwas wie Andromaques Ersatzmann. Nur aufgrund dieser fundamentalen Janusköpfigkeit kann Astyanax überhaupt als Zahlungsmittel fungieren. Bezeichnenderweise

12 Vgl. Ebda., I, 4. 
droht die Transaktion genau dann zu scheitern, als Pyrrhus in vollem Umfang die andere Seite der Münze zu sehen bekommt.

C'est Hector, (disait-elle en l'embrassant toujours;)

Voilà ses yeux, sa bouche, et déjà son audace,

C'est lui-même, c'est toi, cher Epoux, que j'embrasse. ${ }^{13}$

Intim zu tauschen versucht nicht nur Pyrrhus, sondern im Gegenzug auch Andromaque. In dem Maße, wie sie sich zur Heirat gezwungen sieht, verfällt sie auf den Plan, Pyrrhus zwar zu heiraten, unmittelbar im Anschluss daran jedoch Selbstmord zu begehen. Da Pyrrhus sie liebt, so ihre Erwägung, wird er ihren Sohn lieben und am Leben erhalten. Indem sie sich ihm gibt, gibt sie ihm den Sohn und entzieht sich selbst. Wir haben es in beiden Tauschrelationen mit einem komplementären Spiel von Geben und Verweigern intimer Tauschwerte zu tun. Pyrrhus nimmt Andromaque ihren Sohn und gibt ihn ihr zurück, sofern sie ihn heiratet. Andromaque willigt ein, Pyrrhus zu heiraten, überantwortet ihm damit ihren Sohn und verweigert sich anschließend im Selbstmord. Diese Transaktion ist kaum weniger komplex und mehrstufig vermittelt als die in Corneilles Cinna. Da jedoch leidenschaftliche Liebe nicht tauschbar ist, gibt sie nur, um das, was sie nicht geben kann, sofort zurückzunehmen. Und dennoch ist nicht zu übersehen, dass - wenn denn Andromaques Plan zum Zuge käme - ein Transfer der Gabe Astyanax stattfände. Astyanax wäre nach Abschluss der Transaktion Pyrrhus' (Adoptiv)Sohn. So verworren die Transaktionsbeziehung auch ist, man hätte im Sinne der Gesetze der Verwandtschaft wenn nicht einen Frauentausch, so doch einen Kindertausch vollzogen.

Wie im Folgenden zu zeigen sein wird, weist diese Transaktion nicht unbeträchtliche Gemeinsamkeiten mit Rousseaus Julie, ou La Nouvelle Héloïse auf. Hier wie dort dient der tatsächliche bzw. geplante Tod der Mutter funktionell dazu, deren Kinder an einen anderen Mann weiterzureichen. Dass hierbei gleichsam die Vorzeichen und Positionen vertauscht sind, ist einzuräumen. Während Andromaque Selbstmord begehen will, weil sie einen Mann heiraten soll, den sie hasst - sie kann die Gräuel vor Troja nicht vergessen -, stirbt die wider ihren Willen mit Wolmar verheiratete Julie, als sie sich in eine Lebensgemeinschaft mit Saint-Preux hineingezwungen sieht, bei der sie nicht ausschließen kann, dass sie sich dem früheren Geliebten noch ein weiteres Mal hingibt.

So prägend die Münze Astyanax für die Andromaque-Handlung ist, so sehr sie selbst noch in die Beziehung zwischen Hermione und Oreste hineinspielt, sie

13 Ebda., II, 5, v. 656-658. 
ist alles andere als repräsentativ für Racines Gesamtwerk. Durchweg bestimmend ist stattdessen der Motivkreis von Undank und Verbrechen, wie er in der Beziehung zwischen Hermione und Oreste zum Austrag kommt. Auch dieser Handlungsstrang ist auf eine Ökonomie der Intimität zu beziehen.

Um mit den Zahlungsmitteln und deren Werthaltigkeit/-haftigkeit zu beginnen: Wie man vielfach angemerkt hat, erhebt Racine in der Andromaque die pastorale Liebeskette zum großen tragischen Thema. ${ }^{14}$ Es handelt sich hierbei nicht bloß um eine Verschiebung auf der Gattungsebene, sie betrifft mindestens ebenso sehr die Kodierung der Affekte und prägt zutiefst deren Ökonomie. Auch wenn Oreste und Pyrrhus nicht müde werden zu beklagen, dass sie der Sklaverei ihrer Leidenschaft unterliegen, Andromaque wäre keine Tragödie, wenn solche Leidenschaften nicht mindestens ebenso werthaltig und heroisch wären wie Corneilles Konflikt von amour et devoir. Auch deshalb kann der Liebende Racines wenn nicht Gegenliebe, so doch Dank für seine Taten einfordern. Er verhält sich hierbei analog und zugleich komplementär zum Corneille'schen Helden. So wie Cinna sich Emilie durch eine heroische Tat zu verdienen gedenkt, so Oreste Hermione durch eine Unzahl von Verbrechen. Geändert hat sich hierbei >lediglich، das Vorzeichen.

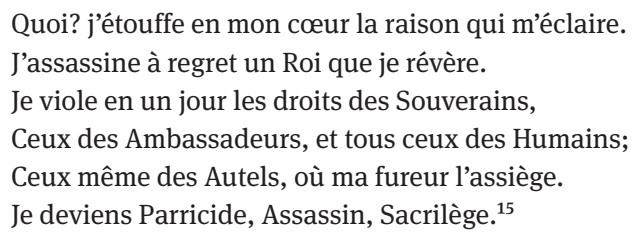

Nicht gesellschaftlich, sondern intim ist dieser Tausch einmal mehr, weil Oreste als Verbrecher Hermione keine gesellschaftliche Gabe zu geben hat. Als Andromaque zum Schein in die Ehe mit Pyrrhus einwilligt, fordert Hermione, die ihrerseits Pyrrhus liebt, aus gekränkter Leidenschaft Oreste auf, sie zu rächen und Pyrrhus bei der Hochzeit zu ermorden. Unter dieser Bedingung, unter der Oreste die oben zitierten multiplen Verbrechen begeht, wäre sie bereit, ihn zu heiraten. Nach begangener Tat jedoch verflucht sie ihn. Er hätte ihren aufflackernden Rachegelüsten nicht nachgeben sollen: „Ah! Fallait-il en croire une Amante insensée? «16 Auch wenn dieser Vorwurf das Moment eines schieren Furors der Leidenschaft in

14 Vgl. grundlegend hierzu Harald Weinrich: Variationen der Liebeskette. In: Ders.: Literatur für Leser. Essays und Aufsätze zur Literaturwissenschaft. München: dtv 1986, S. 50-65.

15 Jean Racine: Andromaque, V, 4, v. 1613-1618.

16 Ebda., V, 3, v. 1585. 
den Vordergrund rückt, ist unter dem Blickwinkel einer Ökonomie der Intimität festzuhalten, dass Hermione nichtsdestoweniger ein Tauschangebot abgegeben und anschließend gebrochen hat.

Diese Tauschstruktur macht so etwas wie den Markenkern der (Racine'schen) Ökonomie der Intimität aus. Sie ist von der der Andromaque-Handlung strikt zu unterscheiden. Während diese mit der Münze Astyanax noch über einen konkreten, qualitativen, vermittelten und deshalb begrenzten Tauschwert verläuft, ist der Gegenwert der Liebe in der Oreste-Handlung rein quantitativ. Es ist eine schiere Häufung aller erdenklichen Verbrechen. Gemessen an der Andromaque-Handlung verhält sich diese Tauschstruktur wie eine hyperinflationäre Währung. Und sie ist dennoch von ihr abzugrenzen. Während man in Währungskrisen den Glauben an die Kaufkraft des Geldes verliert, während dort lediglich die Äquivalenzbeziehungen instabil werden, stellt sich bei Racine die Frage, ob Liebe überhaupt tauschbar ist. Während wir es im einen Fall mit einer Binnenkrise des Systems zu tun haben, haben wir es im anderen Fall mit einer Ausgründung in ein anderes, neues System zu tun - und dennoch kann Racine dieses nicht definieren, ohne sich auf das alte zu beziehen.

So markant Racine die Ökonomie der Intimität im Verbrechen zuspitzt, es gibt einen zweiten Term, der zugleich weicher und radikaler ist: Das Geständnis. Weicher als das Verbrechen ist das Geständnis, weil der Gestehende, anders als der Verbrecher, noch keine Schuld auf sich geladen hat. Er kann deshalb keine Gegenleistung fordern. Gerade deshalb kommt im Geständnis die eigentliche Zumutung einer Ökonomie der Intimität zum Ausdruck. Sie fordert Liebe für Liebe ein. Mit anderen Worten: Der (Tausch)Wert der Liebe - und nichts anderes ist im Geständnis gesetzt - ist ihre Wahrheit. Sie hat am Extrempunkt keinen sachlichen Wert zu geben, kein wiedererstelltes Troja, keine verbrecherischen Taten wie etwa den Königsmord. Konsequent ist diese Zuspitzung auf das Geständnis insofern, als selbst die Verbrechen im Grunde nichts anderes als das Ausmaß und die Wahrheit der Liebe bezeichnen.

$\mathrm{Zu}$ betrachten sind nicht zuletzt vergleichsweise geistesgeschichtliche Rahmenbedingungen. Wie mehrfach geltend gemacht wurde, setzt Affektökonomie voraus, dass in der Moderne niedere Affekte wie Habsucht und Eigennutz nicht länger streng sanktioniert werden. Man kann dasselbe für die Ökonomie der Intimität unterstellen, wenn auch mit gegenläufigem Ergebnis. Während - Adam Smith zufolge - in einem System des Warentauschs, weil es dinglich vermittelt ist, trotz der Habsucht des Einzelnen allgemeine Wohlfahrt entstehen kann, weist die Ökonomie der Intimität bei Racine eine grundsätzlich andere Dynamik auf. Sie endet in einer verhängnisvollen Kettenstruktur. In diesem Sinne ist der Zirkulation der Waren die Liebeskette der Andromaque entgegenzusetzen. In ihr schließt sich kein Kreis, sie bildet stattdessen eine offene Verweisstruktur, deren 
Ausgangspunkt der Tod ist und die nur im Tod zur Ruhe kommen kann. Damit ist neben den monströsen Gaben der Liebe und neben der Gabe der Wahrheit/ des Geständnisses ein weiteres Merkmal der Ökonomie der Intimität benannt. Sie ist hochgradig volatil, sie oszilliert im steten Wechsel um die Pole von Liebe und Hass. Wie präzise diese Volatilität an Tauschmodelle gekoppelt ist und sich nicht bloß Stimmungsschwankungen verdankt, zeigt sich, wenn man die Andromaque-Handlung mit der Oreste-Handlung vergleicht. Wenn der Plan Andromaques zur Ausführung käme, würde die tragische Entwicklung nach vollzogenem Tausch - Pyrrhus erhielte zwar nicht Andromaque selbst, aber doch ihren Sohn in einen Ruhepunkt einmünden. Anders gelagert ist die Beziehung zwischen Oreste und Hermione. Da sie kein Drittes haben, mittels dessen sie tauschen könnten, trennen sie sich sunverrichteter Dingeı. Hermione begeht an der Leiche des Pyrrhus Selbstmord, den ohnmächtigen Oreste entfernen seine Gefährten. Während sich im Fall von Andromaques Selbstmord die Handlung schlösse, weil es mit Astyanax noch einen Tauschwert gibt, mit dessen Transfer die Handlung zu einem mehr oder minder guten Abschluss kommen könnte, bricht im tragischen Finale der Oreste-Handlung die ganze Polarität der Beziehung, ihre ganze volatile Schwankungsbreite noch einmal auf.

\section{Rousseau: Les confessions}

So wie die Affektökonomie erst im 18. Jahrhundert voll ausgeprägt ist, so auch die Ökonomie der Intimität. Man versucht sie sozial zu vermitteln, teilweise zu entschärfen - man denke an Philines Satz aus dem Wilhelm Meister: »und wenn ich dich lieb habe, was geht's dich an? « ${ }^{17}$-, aber auch auszuweiten. Sie betrifft nicht mehr bloß die Beziehung zwischen Sexualpartnern, sondern auch die zwischen Freunden - und selbst noch die zum Publikum.

Das autobiographische Werk Rousseaus legt von Letzterem ein breites Zeugnis ab. Nachdem Rousseau öffentlich in die Kritik geraten ist, verteidigt er sich nicht mit spitzer Feder, sondern verfällt auf die etwas überraschende Idee, so etwas wie eine Lebensbeichte abzulegen. Statt sich ins Recht zu setzen, setzt er sich ins Unrecht, statt sich zu verteidigen, gesteht er wie sonst nur der leidenschaftlich Liebende bei Racine. Es ist durchaus einzuräumen, dass die Rousseau'schen und die Racine'schen Geständnisse nicht in jeder Hinsicht deckungsgleich

17 Johann Wolfgang von Goethe: Wilhelm Meisters Lehrjahre. Hamburger Ausgabe. Bd.7. Herausgegeben von Erich Trunz. München: dtv 1982, S.7-610; hier: S. 235. 
sind. Während der Racine’sche Held seine Liebe gesteht und Gegenliebe fordert, gesteht der Autor Rousseau in den Confessions sein Leben, um dem zu entgehen, was er seine Verfolgungen nennt. Im einen Fall reden wir über eine leidenschaftliche Liebe, die alle Normen sprengt, im anderen Fall über eine Autorschaft, die unter Umgehung literarischer Kritik, unter Zurückweisung der Anfeindungen, die eine öffentliche Person wie Rousseau zu gewärtigen hat, eine intime, da freundschaftliche Beziehung zum Publikum aufzubauen versucht. So groß die Unterschiede im Einzelnen auch sind - hier wie dort versucht man Wahrheit gegen Liebe/Freundschaft unter Ausschluss gesellschaftlicher Normen und Instanzen zu tauschen. Hier wie dort ist deshalb die >Gabe ‘ der Wahrheit unlimitiert. So wie der Racine'sche Held alle erdenklichen Verbrechen begeht, um die Wahrheit seiner Liebe zu beweisen, so häufen sich in den Confessions in zunehmendem Maß die Akten, um so ein Projekt ohne Ende zu eröffnen.

Das Konzept einer intimen, da außergesellschaftlichen Beziehung zum Publikum datiert nicht erst auf die Verfolgungen zurück. Wenn Rousseau in den Confessions anmerkt, es sei ihm nicht möglich, für Geld zu schreiben, da dies seine Freiheit einschränke, ${ }^{18}$ so ging er bereits im Vorwort zur Nouvelle Héloïse noch einen Schritt weiter:

Tout honnête homme doit avouer les livres qu'il publie. Je me nomme donc à la tête de ce recueil, non pour me l'approprier, mais pour en répondre. S'il y a du mal, qu'on me l'impute; s'il y a du bien, je n'entends point m'en faire honneur. Si le livre est mauvais, j'en suis plus obligé de le reconnaître: je ne veux pas passer pour meilleur que je ne suis. ${ }^{19}$

Bekenntnishaft ist also Autorschaft als solche. Rousseau arbeitet diesen Umstand heraus, indem er selbst noch das symbolische Kapital oder die Ehre, die ihm aus seinem Buch zuteilwerden könnte, dem Leser vor die Füße wirft.

18 Vgl. Jean-Jacques Rousseau: Les confessions. In: Ders.: Euvres complètes. Bibliothèque de la Pléiade. Bd.1. Herausgegeben von Bernard Gagnebin/Marcel Raymond. Paris: Gallimard 1959, S.1-656; hier: S. 402.

19 Jean-Jacques Rousseau: Préface [zu Julie, ou La Nouvelle Héloïse]. In: Ders.: Euvres complètes. Bibliothèque de la Pléiade. Bd.2. Herausgegeben von Bernard Gagnebin/Marcel Raymond. Paris: Gallimard 1964, S. 5-6; hier: S. 5. 


\section{Rousseau: Julie ou La Nouvelle Héloïse}

Solche bekenntnishaften, intimen Arbeitsmodelle betreffen nicht nur den >Berufsstand des Schriftstellers, ${ }^{20}$ sondern gleichermaßen den des Pädagogen. Auch wenn dort die Risiken beträchtlich größer sind und weitere Zweideutigkeiten auftreten. So weigert sich Saint-Preux, für das Unterrichten Julies Geld zu nehmen. Man vermutet zu Recht: um ihr sebenbürtigı zu sein. Saint-Preux denkt diesen Gedanken in nachgerade zynischer Weise zu Ende: Wenn er sich seine Lehrtätigkeit bezahlen lässt, ist er dem Hausherrn verpflichtet und kann die Tochter nicht verführen. Wenn er kein Geld nimmt, stehen ihm alle Optionen offen. Im Gefolge dieser Erwägungen hat Saint-Preux ein Verhältnis mit Julie. Man verweist ihn des Hauses. Er kehrt nach langer Trennung zurück - man trägt ihm an, zum Erzieher der Kinder seiner ehemaligen Geliebten zu werden. Der Roman lässt sich vor diesem Hintergrund wie eine gewaltige Transaktion - durchaus im Sinne von geschäftlicher Transaktion - lesen, die es auf vielen Umwegen ermöglicht, dass ein (bürgerlicher) >Hausfreund $\mathrm{zu}$ einem `Erzieher ohne Bezahlung` wird. Der gesamte Handlungsbogen der Nouvelle Héloïse ist wie maßgeschneidert auf diese Transaktion zugeschnitten. Nachdem Saint-Preux im ersten Erziehungsprojekt sich in seine Schülerin verliebt hat, soll er im zweiten in einer Form von Affektübertragung deren Söhne lieben. Er soll sie lieben, da sie sie liebt, und die Söhne statt der Mutter lieben. Da nach den Kodes einer wahren, und das heißt immer auch: einer unabdingbaren Liebe Affekte nicht vermittelbar sind, ist das Modell dieser Gefühlsübertragung, dieses Tauschhandels mit Gefühlen im Sinne einer Ökonomie der Intimität zwar systemkonform, aber dennoch nicht lebbar. Es ist deshalb kein Zufall, dass Julie wie aufgrund eines unglücklichen Zufalls stirbt.

Mit Blick auf eine Ökonomie der Intimität/des Affekts kommt dem Tod Julies eine nicht zu unterschätzende Bedeutung zu. Im Vorfeld ist festzuhalten, dass diese Ökonomie weder an Modellen einer aufkeimenden Nationalökonomie, an Modellen von Geld- und Hauswirtschaft abgelesen ist noch an deren Kalkulierbarkeit, wie Vogl dies in Kalkül und Leidenschaft unterstellt. Stattdessen bewegen wir uns im Umfeld eines neuen pädagogisch-medizinischen Wissens. Es ist einzuräumen, dass es zwischen beiden Wissensbereichen in epistemologischer Hinsicht Überschneidungen gibt. So verweist Vogl darauf, dass das Bewässerungssystem im Garten von Clarens an Harveys System des Blutkreislaufs abgelesen

20 Vgl. hierzu Franziska Sick: Berufung ohne Ruf. Berufslegenden bei Jean-Jacques Rousseau und Michel Leiris. In: Patricia Oster/Karlheinz Stierle (Hg.): Legenden der Berufung. Heidelberg: Winter 2012, S.127-149; hier: S. 134-143. 
ist. ${ }^{21}$ Natur wird regulierbar, indem man auf sanfte Weise ihre Energien umlenkt. In diesem Sinne - so ließe sich im Anschluss an Vogl argumentieren - stellt das elysische Projekt die Blaupause noch für das erotisch-pädagogische dar. So wie man dort die Wasserkräfte umzulenken versucht, so hier die Triebkräfte einer leidenschaftlichen Liebe. Wie Julie weiß, lassen sich so jedoch nicht alle Triebkräfte bändigen. Auch wenn Saint-Preux ihre Kinder liebt, so liebt er doch nach wie vor sie selbst. Um diese affektive Bindung in den Griff zu bekommen, schlägt Julie Saint-Preux eine Heirat mit ihrer Cousine Claire vor. Beide lehnen ab. Julie muss zuvörderst auch deshalb sterben, da sich die Leidenschaft Saint-Preux‘ als unregulierbar erweist.

Womit sich unter der Hand zeigt, dass das Konzept der Affektökonomie, dass das »Kalkül der Leidenschaft« (Vogl) scheitert und nur noch durch eine Ökonomie der Intimität stabilisiert werden kann. Zum Zuge kommt hierbei eine Tauschökonomie, auf die wir in ähnlicher Form bereits beim frühen Racine, in der Andromaque-Handlung, gestoßen sind. In beiden Fällen wird mit dem Tod der Mutter die Gabe der Frau durch die Gabe der Kinder ersetzt. Es ist hierbei nicht auszublenden, dass beide Tauschtransaktionen, obwohl sie auf der Ebene der verwandtschaftlich-geschlechtlichen Tauschbeziehung strukturgleich sind, auf einer zweiten, sachlichen Tauschebene äußerst divergent kodiert sind. Während Andromaque versucht, durch ihren Selbstmord ihrem Sohn Asyl zu erwirken, dient der Tod Julies dazu, einen Erzieher als Kinderfreund zu installieren. In Blick zu nehmen ist ferner eine nicht unbeträchtliche gegenläufige historische Verschränkung. Wie bereits dargelegt: Die Andromaque-Handlung ist noch vergleichsweise traditionell gefasst. Sie besitzt mit Astyanax noch einen dinglichen, familiär kodierten Tauschwert. Der Umstand, dass Rousseau auf eben dieses frühe Transaktionsmodell zurückgreift, zeigt, wie sehr es ihm darum zu tun ist, das schiere >Wüten Racince'scher Leidenschaft sozial rückzuvermitteln. Es zeigt sich auch daran, dass Rousseau das Kernnarrativ Racines umerzählt. Typischer Weise bricht bei Racine ein ungeliebter Dritter in eine bestehende Liebesbeziehung ein. Nachdem er alle erdenklichen Gesetze gebrochen hat, ermordet er seinen Konkurrenten oder aber betreibt den Tod der Geliebten. Gänzlich anders ist das Personal in der Nouvelle Héloïse aufgestellt. Hier sind die Liebenden nicht länger von einem rasenden Liebhaber bedroht, sondern von Normen. ${ }^{22}$ In Gang gesetzt wird die Entwicklung damit durch einen verhinderten Tausch und nicht durch

21 Vgl. Joseph Vogl: Kalkül und Leidenschaft, S. 230-238.

22 In Blick zu nehmen ist hierbei nicht nur das Verdikt von Julies adelsstolzem Vater, sondern auch die Rücksichtnahme Claires gegenüber ihrer Freundin und nicht zuletzt die gesellschaftliche Rücksicht, derer sich Edouard im Falle Julies befleißigt. 
die Unfähigkeit zu tauschen. Gänzlich anders ist nicht zuletzt die Affektlage der Liebenden kodiert. Während bei Racine der Liebende in selbstbezüglicher Weise dem Furor seiner Leidenschaft verfällt, veredeln Julie und Saint-Preux ihre Liebe zu einer freundschaftlichen Beziehung. Unter der Hand verändert sich hierbei die Grundgeometrie von Liebe und Leidenschaft. Während sie bei Racine direkt und rücksichtslos besitzergreifend ist, wird sie bei Rousseau zu einem zutiefst vermittelten Gefühl. Der Liebende will den Geliebten nicht besitzen, sondern - so zumindest lautet die Ideologie dieses Liebeskonzepts - er will, dass der Andere glücklich ist.

Auch wenn die empathische Liebe Rousseaus zutiefst vergesellschaftet ist, ist sie kaum weniger dereguliert als die Racine'sche. Sie wird zum einen den Racine'schen Bodensatz leidenschaftlicher Liebe nicht los. Wahr ist Liebe - auch bei Rousseau - nur als leidenschaftliche. Und sie verfängt sich zum anderen dort, wo sie die Leidenschaft mit empathischen Mitteln bricht, in einem Spiegelkabinett wechselseitiger Rücksichtnahmen. Dem obstinaten Vorwurf des ingrat bei Racine ${ }^{23}$ steht bei Rousseau eine elaborierte Buchhaltung von Liebesschulden gegenüber, die nichtsdestoweniger zu keiner stabilen Tausch- und Beziehungsstruktur führt.

Zumal zu Beginn des Romans kodiert sich die Ökonomie der Liebe nach Maßgabe einer Bilanzierung von Liebesleid und -schuld. So hält Saint-Preux Julie vor, nachdem sie ihre Beziehung aus gesellschaftlichen Gründen einschränken mussten, dass sie doch sehr heiter sei. Seiner Auffassung nach müsse sie hierunter genauso viel leiden wie er. Wenn nicht - das ist sein Vorhalt -, liebe sie ihn weniger als er sie. Im Sinne der empathischen Liebe wendet Julie ein: »La singulière marque d'attachement, que de vous plaindre de ma santé! « ${ }^{24}$ Man sieht, wie vertrackt diese Tauschordnung ist. Der eigentliche Tauschwert wäre leidenschaftlicher Ausdruck von Liebesleid bis hin zur Krankheit, aber diesen darf man aus Gründen der empathischen Liebe nicht einfordern.

Überlagert wird diese Beziehung durch das Konzept einer ehrenhaften Liebe, die zumindest im Grundansatz an Corneille erinnert. Zu lieben ist der Geliebte, heiratbar ist er nur, insofern er ehrenhaft, und das heißt im Falle von Julie und Saint-Preux zuvörderst, insofern er tugendhaft ist. ${ }^{25}$ Anders als das Corneille'sche

23 Vgl. Erich Köhler: >Ingrat` im Theater Racines. Über den Nutzen des Schlüsselworts für eine historisch-soziologische Literaturwissenschaft. In: Ders.: Vermittlungen. München: Fink 1976, S. 203-218.

24 Vgl. Jean-Jacques Rousseau: Julie, ou La Nouvelle Héloïse. In: Ders.: Euvres complètes. Bibliothèque de la Pléiade. Bd. 2. Herausgegeben von Bernard Gagnebin und Marcel Raymond. Paris: Gallimard 1964, S.1-745; hier: S. 49.

25 Es wäre näher darzulegen, in wie umfangreicher Weise Rousseau in La Nouvelle Hélö̈se den Gegensatz von innerer und äußerer Ehre ausarbeitet. 
Ehrkonzept ist das Rousseau'sche jedoch nicht gesellschaftlich kodiert. Es ist so innerlich wie zugleich privat-intim. Ehrkonzepte, Werte- und Tauschordnungen bedürfen jedoch regelmäßig einer dritten Instanz, die die Einhaltung der Wertordnung überwacht, objektiviert und sanktioniert. Ohne sie bleiben sie kraftlos. Das zeigt sich exemplarisch am Verhältnis von Julie und Saint-Preux. Saint-Preux hat Julie in Vevey zweimal verführt. Nach Maßgabe ihres Ehrbegriffs hätte sie ihn bereits nach der ersten Verführung endgültig verstoßen müssen. Als sie in Clarens ein drittes Mal in die Situation der Verführung gerät, stirbt sie. An dieser Stelle wird kenntlich, wie fragil die moderne Ökonomie der Intimität ist. Wenn die Liebenden selbst über den inneren Wert des Partners entscheiden, sind sie in den Fall gesetzt, dass sie wie eine Notenbank den Kurs der Währung festsetzen, um zugleich als Liebende mit ihr zu bezahlen.

Relative Stabilität gewinnt diese Tauschstruktur, insofern sie in den Lebensund Todesschulden einen Absolutwert oder aber auch so etwas wie eine harte Währung besitzt. Das ist zu Teilen ein durchaus traditionelles Register. Kulturell verbreitet ist die Vorstellung, dass man den Eltern zu unendlichem Dank verpflichtet ist, weil man ihnen das eigene Leben schuldet. ${ }^{26}$ Rousseau doppelt diese Beziehung auf, indem er die Lebensschuld mit der Todesschuld verbindet. Es ist neben dem Kniefall des Vaters der Tod der Mutter, den vorgeblich Julie durch ihren Fehltritt verursachte, der sie in die Ehe mit Wolmar zwingt. Es ist mit ihrem Tod ihr letzter, postumer Brief vom Sterbebett, der Saint-Preux zur Erziehung ihrer Kinder verpflichtet. Die Hochzeit mit Wolmar, die Erziehung der Kinder, alle relevanten Transaktionen des Romans werden durch den Tod vermittelt. Darüber hinaus durchzieht der Tod den Roman wie ein roter Faden. Man denke an die Krankheit(en) Julies, an Saint-Preux' Traum von der toten Julie oder daran, dass der Tod immer dann, wenn eine Trennung ansteht, als Verhandlungsmasse und Münze ins Spiel gebracht wird. Das Spektrum reicht von Selbstmorddrohungen bis zu der schwächeren Behauptung, dass man die Trennung nicht überleben werde. Blockiert wird diese Tauschbeziehung regelmäßig durch das Register der empathischen Liebe. So sehr man die Trennung mit der Münze des eigenen Todes quittieren will - man kann diese Münze nur vorzeigen, aber nicht in Umlauf bringen, weil der eigene Tod für den Geliebten zu schmerzvoll wäre. Auch deshalb muss der Tod von Julie und Julies Mutter zufällig sein. Er ist die letzte und härteste Währung einer Ökonomie der Intimität, die zugleich nicht handelbar ist. In anderer Form kehrt hierbei das Aporetische dieser Ökonomie wieder: So funktio-

26 Vgl. David Graeber: Schulden, S. 98. 
nal notwendig der Tod als Tauschwert in ihr ist, so wenig ist er systemimmanent zu begründen. ${ }^{27}$ Auch deshalb tritt er als externer Zufall auf.

Wie wir bisher gesehen haben: La Nouvelle Hélö̈se vollzieht in der Gesamtanlage eine Transaktion, in der die Geliebte gegen die Kinder getauscht wird. Grundiert und begleitet ist diese Transaktion von Motiven empathischer und ehrenhafter Liebe sowie von Todes- und Lebensschulden. Neben diesen unmittelbar tauschrelevanten, da interaktiven Strukturen sind abschließend die interpersonellen Strukturen, Verwandtschaftsbeziehungen zu betrachten. Sie sind, anders als in traditionellen Systemen, nicht länger unmittelbar tauschrelevant, bilden aber dennoch so etwas wie eine Plattform, auf der die jeweiligen Tauschtransaktionen prozessiert werden können.

So sehr auch Rousseau die Beziehung zwischen Liebenden und Freunden als eine außergesellschaftliche Beziehung zwischen zweien propagiert, so unübersehbar ist, dass sie stets von einem Dritten, von einem Vermittler begleitet, befördert und ansatzweise reguliert wird. Das beginnt bereits mit dem Kuss, den SaintPreux Julie gibt - er küsst zum Scherz zunächst Claire, dann erst Julie -, und das setzt sich in vergleichbarer Weise in der gesamten Beziehungsgeometrie des Romans fort. Zumal Saint-Preux ist nachgerade von Vermittlerfiguren umstellt. Claire, Edouard und selbst Wolmar - sie alle versuchen, ihm den rechten Weg zu weisen. Wobei diese Vermittler, obwohl sie formal die Position des Dritten einnehmen, sachhaltig keine starke, normsetzende Position beziehen. Deutlich wird dies bereits bei Claire, die sich mit dem Satz »Tu verras, tu verras ce que c'est qu'une Duégne de dix-huit ans! ${ }^{28}$ in die Beziehung zwischen Julie und SaintPreux einführt. Kaum weniger ambig ist der Vermittler Edouard. Er ist zum einen selbst in Julie verliebt und hat andererseits Affären in Rom, die kaum weniger problematisch sind als die Saint-Preux'sche. In aller Deutlichkeit zeigt sich hierbei, dass und wie sehr einer Ökonomie der Intimität die regulierende Position des Dritten fehlt. Sie versucht sie nachzubilden, indem sie den sich Liebenden einen Freund beigesellt, aber damit ist kaum mehr gesetzt als eine zweite dyadische, da intime Beziehung. Zwei verschränkte dyadische Beziehungen ergeben jedoch keine triadische Struktur, sie bilden allenfalls einen ménage à trois mit volatiler Affektökonomie.

27 Auch diese Systemnotwendigkeit exponiert bereits Racine. In Andromaque kündigt Andromaque gleich eingangs gegenüber Pyrrhus ihren Selbstmord an, als dieser ihr mit der Ermordung des Astyanax droht. In Bérénice setzen Bérénice, Titus und Antiochus reihum die Münze des Selbstmords ein, bevor Bérénice am Ende zugunsten des Lebens, wenn auch zum allgemeinen Leid alle zur Entsagung anhält.

28 Jean-Jacques Rousseau: Julie, ou La Nouvelle Héloïse, S. 46. 
Nicht zuletzt trägt dieses Beziehungsgeflecht sich in Familienstrukturen ab. Einerseits rekodiert Rousseau die Liebesbeziehung mit familialen Registern - so sagt Julie zu Saint-Preux, sie fühle sich wie seine Schwester, und er seinerseits behauptet, mit ihr vor der Ehe zu schlafen sei für ihn wie eine Blutschande. ${ }^{29} \mathrm{Zum}$ Anderen prägt der Roman durchaus neuartige Familienstrukturen aus. So bildet die Gesellschaft von Clarens zum Ende des Romans eine großfamiliale Hausgemeinschaft - mit wie loser familialer Bindung auch immer. Vollends wenn SaintPreux Claire heiraten würde, würde er als der Geliebte Julies ihre beste Freundin heiraten. Das wäre eine Hochzeit unter Freunden, vermittelt durch Freunde. In ihr wäre es die Geliebte und nicht länger der Vater, der über die Gabe der Geliebten verfügt. So besehen, begründet die Ökonomie der Intimität im Grundansatz neue Familienstrukturen. Nicht anzutreffen sind solche fundamentalen Verschiebungen bei Racine. Da es ihm darum zu tun ist, Leidenschaft, Intimität allererst als Wert zu setzen, entwickelt er den Wert innerhalb der traditionellen Verwandtschaftskodes. Die Liebenden brechen deren Gesetze bis hin zum Inzest (Phèdre). Gesetzt ist damit noch keine neue Struktur, sondern ein schierer Gegenwert. Von einer vollwertig entwickelten Ökonomie der Intimität ist erst bei Rousseau zu reden, weil erst bei ihm ein hinlänglich differenziertes Arsenal von Vermittlungsstrukturen zu verzeichnen ist.

Da die Racine'sche Position rein negativ ist, erscheint es lohnender, die Rousseau'sche Heiratspolitik mit der Corneille'schen zu vergleichen. Die Differenzen sind durchaus markant. Während Augustus die Clans eliminiert, um ein vertikales System des Frauentauschs zu errichten, eliminiert Rousseau das System der Clans zugunsten einer horizontalen, man könnte auch sagen: ansatzweise demokratischen Struktur. Hier wie dort lässt sich eine geänderte Ökonomie der Sexualität darüber beschreiben, dass die Gabe der Frau/des Geliebten in andere Hände gelangt. Sie gelangt bei Corneille in die Hände des Souveräns. Sie gelangt bei Rousseau tendenziell, sofern Liebe tauschbar wäre, in die Hände der Freunde, der Gleichaltrigen. In wie loser Weise auch immer ist diese Verschiebung bis heute wirksam.

29 Vgl. Ebda., S. 42: »Je frémirois de porter la main sur tes chastes attraits, plus que du plus vil inceste, et tu n'es pas dans une sûreté plus inviolable avec ton père qu'avec ton amant." 


\section{Bibliographie}

Bateson, Gregory: Ökologie des Geistes. Anthropologische, psychologische, biologische und epistemologische Perspektiven. Übersetzt von Hans Günter Holl. Frankfurt a. M.: Suhrkamp (1981) ${ }^{5} 1983$.

Baudrillard, Jean: L'échange symbolique et la mort. Paris: Gallimard 1976.

Corneille, Pierre: Cinna ou la clémence d'Auguste. In: Ders.: Euvres complètes. Bibliothèque de la Pléiade. Bd.1. Herausgegeben von Georges Couton. Paris: Gallimard 1980, S.903-969.

Goethe, Johann Wolfgang von: Wilhelm Meisters Lehrjahre. Hamburger Ausgabe. Bd.7. Herausgegeben von Erich Trunz. München: dtv 1982, S.7-610.

Graeber, David: Schulden. Die ersten 5.000 Jahre. Aus dem Amerikanischen von Ursel Schäfer/ Hans Freundl u. a.. Stuttgart: Klett-Cotta ('2011) ${ }^{7} 2012$.

Köhler, Erich: ১Ingrat، im Theater Racines. Über den Nutzen des Schlüsselworts für eine historisch-soziologische Literaturwissenschaft. In: Ders.: Vermittlungen. München: Fink 1976, S. 203-218.

Lévi-Strauss, Claude: Les structures élémentaires de la parenté. Berlin/New York: Mouton de Gruyter (11949) ${ }^{2} 1967$.

Luhmann, Niklas: Soziale Systeme. Grundriss einer allgemeinen Theorie. Frankfurt a. M.: Suhrkamp 1984.

-: Liebe als Passion. Zur Codierung von Intimität. Frankfurt a. M.: Suhrkamp (1982) ${ }^{13} 2015$.

-: Liebe. Eine Übung. Herausgegeben von André Kieserling. Frankfurt a. M.: Suhrkamp (12008) ${ }^{4} 2014$.

Mauss, Marcel: Essai sur le don. Forme et raison de l'échange dans les sociétés archaïques. Paris: PUF (1950) ${ }^{2} 2012$.

Racine, Jean: Andromaque. In: Ders.: Eruvres complètes. Bibliothèque de la Pléiade. Bd.1. Herausgegeben von Georges Forestier. Paris: Gallimard 1999, S. 193-256.

Rousseau, Jean-Jacques: Les confessions. In: Ders.: Fuvres complètes. Bibliothèque de la Pléiade. Bd.1. Herausgegeben von Bernard Gagnebin/Marcel Raymond. Paris: Gallimard 1959, S.1-656.

-: Julie, ou La Nouvelle Héloïse. In: Ders.: Fuvres complètes. Bibliothèque de la Pléiade. Bd. 2. Herausgegeben von Bernard Gagnebin/Marcel Raymond. Paris: Gallimard 1964, S.1-745.

Sick, Franziska: Tragisches Potential und untragisches Ende. Absolutistische Konzepte in den frühen Dramen Corneilles. In: Rudolf Behrens/Roland Galle (Hg.): Historische Anthropologie und Literatur. Romanistische Beiträge zu einem neuen Paradigma der Literaturwissenschaft. Würzburg: Königshausen \& Neumann 1995, S. 91-107.

-: Berufung ohne Ruf. Berufslegenden bei Jean-Jacques Rousseau und Michel Leiris. In: Patricia Oster/Karlheinz Stierle (Hg.): Legenden der Berufung. Heidelberg: Winter 2012, S. 127-149.

Vogl, Joseph: Kalkül und Leidenschaft. Poetik des ökonomischen Menschen. Zürich: Diaphanes (12002) ${ }^{4} 2011$.

Weinrich, Harald: Variationen der Liebeskette. In: Ders.: Literatur für Leser. Essays und Aufsätze zur Literaturwissenschaft. München: dtv 1986, S. 50-65. 